E D I T O R I A L S

\title{
Treatment of Malaria in Pregnancy
}

\author{
Joel Tarning, Ph.D.
}

Malaria during pregnancy is a major public health concern and an important contributor to maternal and infant morbidity and mortality in malaria-endemic countries. ${ }^{1}$ Pregnant women are particularly susceptible to malaria, and in lowtransmission settings they have a greater risk of severe Plasmodium falciparum malaria. P. falciparuminfected red cells sequester in the placenta, disrupting nutritional exchange between mother and fetus and causing intrauterine growth retardation. Malaria is associated with an increased risk of abortion, stillbirth, and low birth weight. ${ }^{2}$

The World Health Organization (WHO) now recommends that all women in the second or third trimester of pregnancy who have uncomplicated P. falciparum malaria should be treated with artemisinin-based combination therapy. ${ }^{3}$ The short-acting but potent artemisinin component (i.e., artemether, artesunate, or dihydroartemisinin) reduces the number of parasites substantially during the first 3 days of treatment. The longer-acting partner drug (i.e., lumefantrine, piperaquine, amodiaquine, or mefloquine) eliminates the remaining parasites, thereby preventing recrudescent malaria. The longer-acting partner drug is also responsible for the posttreatment prophylactic effect, which prevents new infections while drug concentrations in blood exceed the minimum inhibitory concentration of the parasite. Thus, the duration of post-treatment prophylactic effect is a consequence of the potency and the elimination half-life of the drug. The same mechanism of action is used in intermittent preventive treatment, in which repeated curative antimalarial treatments eliminate potential asymptomatic infections and also prevent new infections. However, artemisinin-based combination therapy is not currently recommended for intermittent preventive treatment in pregnancy. The current recommendation from the WHO is for all women in malaria-endemic areas in Africa to receive intermittent preventive treatment with sulfadoxine-pyrimethamine as part of their antenatal care. ${ }^{3}$ Unfortunately, the effectiveness of sulfadoxine-pyrimethamine is challenged by widespread drug resistance in many areas.

However, information on the safety, efficacy, and pharmacokinetics of artemisinin-based combination therapies in pregnant women is limited. Two articles in this issue of the Journal, by the PREGACT Study Group ${ }^{4}$ and Kakuru et al., ${ }^{5}$ present new findings to support the use of artemisinin-based combination therapy in both the prevention and the treatment of uncomplicated $P$. falciparum malaria in pregnancy.

In the PREGACT trial, involving 3428 pregnant women with uncomplicated P. falciparum malaria in four African countries (Burkina Faso, Ghana, Malawi, and Zambia), cure rates of 94.8 to $99.2 \%$ were achieved after treatment with four different antimalarial drug combinations (artemether-lumefantrine, amodiaquine-artesunate, mefloquine-artesunate, and dihydroartemisininpiperaquine). These data provide needed evidence that artemisinin-based combination therapies are effective in pregnant women with malaria in Africa, without evident safety concerns. Drugrelated adverse events in mothers were transient and relatively mild in all treatment groups. Two combinations, dihydroartemisinin-piperaquine and artemether-lumefantrine, had better safety and side-effect profiles. The rates of placental malaria infection were similar among the treatment groups, and approximately $15 \%$ of delivered babies had low birth weight. As expected, a substantially shorter post-treatment prophylactic effect was seen in the artemether-lumefantrine group because of the relatively shorter elimination half-life of lumefantrine ${ }^{6}$ as compared with the other partner drugs. ${ }^{7}$ This may be of particular importance in high-transmission settings, where a prolonged post-treatment prophylactic 
effect should reduce the overall morbidity due to malaria by reducing the frequency of new infections. Among the four drug combinations studied, dihydroartemisinin-piperaquine had the best efficacy and an acceptable safety profile, with an additional benefit of a longer post-treatment prophylactic effect, which supports its suitability as a chemoprophylaxis or chemoprevention agent.

The trial conducted by Kakuru et al. demonstrates the safety and efficacy of dihydroartemisinin-piperaquine as intermittent preventive treatment for malaria in pregnant women in Uganda. A total of 300 pregnant women received either three treatments of sulfadoxine-pyrimethamine, three treatments of dihydroartemisininpiperaquine, or monthly treatment with dihydroartemisinin-piperaquine during pregnancy. The prevalence of histopathologically confirmed placental malaria was significantly higher after sulfadoxine-pyrimethamine treatment $(50 \%)$ than it was after three treatments of dihydroartemisinin-piperaquine $(34 \%)$ or after monthly treatment with dihydroartemisinin-piperaquine (27\%). The rate of adverse birth outcomes after monthly dihydroartemisinin-piperaquine treatment was half of that seen in the other treatment groups, which shows the benefit of effective prevention of malaria during pregnancy. Similarly, the incidence of symptomatic malaria and the prevalence of parasitemia among pregnant women were substantially higher in the sulfadoxinepyrimethamine group than in either dihydroartemisinin-piperaquine group; the difference between the sulfadoxine-pyrimethamine group and the monthly dihydroartemisinin-piperaquine group was especially pronounced.

These studies indicate the effectiveness in pregnancy of artemisinin-based combination therapy for the treatment of uncomplicated $P$. falciparum malaria and the effectiveness of dihydroartemisinin-piperaquine for the prevention of malaria, without evident safety concerns. However, the most effective dosing of artemisininbased combination therapies in pregnant women is still debated; studies have shown substantially lower drug concentrations of artemisinin ${ }^{7}$ and partner drugs ${ }^{6}$ in pregnant women than in nonpregnant women. Prospective pharmacokinetic studies involving pregnant women and nonpregnant controls are needed to characterize the pharmacologic properties of these antimalarial drugs in order to improve treatment. New drugs in development are still several years away from clinical use, and evidence-based dosing of currently available antimalarial drugs might increase their therapeutic lifespan by reducing the risk of treatment failures and the development of resistance. This might be particularly important in Southeast Asia, where acquired immunity is lower and resistance to artemisinin and its partner drugs is emerging and spreading. ${ }^{8,9}$

Disclosure forms provided by the author are available with the full text of this article at NEJM.org.

From the Centre for Tropical Medicine and Global Health, Nuffield Department of Clinical Medicine, University of Oxford, Oxford, United Kingdom, and the Mahidol-Oxford Tropical Medicine Research Unit, Faculty of Tropical Medicine, Mahidol University, Bangkok, Thailand.

1. World malaria report 2015. Geneva: World Health Organization (http://www.who.int/malaria/publications/world-malaria -report-2015/en/).

2. McGready R, Lee SJ, Wiladphaingern J, et al. Adverse effects of falciparum and vivax malaria and the safety of antimalarial treatment in early pregnancy: a population-based study. Lancet Infect Dis 2012;12:388-96.

3. Guidelines for the treatment of malaria, third edition. Geneva: World Health Organization, 2015 (http://www.who.int/malaria/ publications/atoz/9789241549127/en/).

4. The PREGACT Study Group. Four artemisinin-based treatments in African pregnant women with malaria. N Engl J Med 2016;374:913-27.

5. Kakuru A, Jagannathan P, Muhindo MK, et al. Dihydroartemisinin-piperaquine for the prevention of malaria in pregnancy. N Engl J Med 2016;374:928-39.

6. Kloprogge F, Piola P, Dhorda M, et al. Population pharmacokinetics of lumefantrine in pregnant and nonpregnant women with uncomplicated Plasmodium falciparum malaria in Uganda. CPT Pharmacometrics Syst Pharmacol 2013;2:e83.

7. Tarning J, Rijken MJ, McGready R, et al. Population pharmacokinetics of dihydroartemisinin and piperaquine in pregnant and nonpregnant women with uncomplicated malaria. Antimicrob Agents Chemother 2012;56:1997-2007.

8. Ashley EA, Dhorda M, Fairhurst RM, et al. Spread of artemisinin resistance in Plasmodium falciparum malaria. $\mathrm{N}$ Engl J Med 2014;371:411-23.

9. Amaratunga $C$, Lim $P$, Suon $S$, et al. Dihydroartemisininpiperaquine resistance in Plasmodium falciparum malaria in Cambodia: a multisite prospective cohort study. Lancet Infect Dis 2016 January 7 (Epub ahead of print).

DOI: 10.1056/NEJMe1601193

Copyright (C) 2016 Massachusetts Medical Society.

\title{
HLA-Incompatible Kidney Transplantation — Worth the Risk?
}

\author{
Lionel P.E. Rostaing, M.D., Ph.D., and Paolo Malvezzi, M.D.
}

Chronic kidney diseases are a major worldwide prevalence of chronic kidney disease is approxisocietal burden. In the United States, where the mately $14 \%,{ }^{1}$ close to 1 million persons have end- 